\title{
Enhancement of particle trapping in the wave-particle interaction
}

\author{
R. Bachelard ${ }^{\mathrm{a}, 1}$, A. Antoniazzi ${ }^{\mathrm{b}}$, C. Chandre ${ }^{\mathrm{a}}$, D. Fanelli ${ }^{\mathrm{b}}$, \\ M. Vittot ${ }^{\mathrm{a}}$ \\ ${ }^{a}$ Centre de Physique Théorique, CNRS Luminy, Case 907, F-13288 Marseille \\ Cedex 9, France ${ }^{2}$ \\ ${ }^{\mathrm{b}}$ Dipartimento di Energetica and CSDC, Università di Firenze, INFN, via S. \\ Marta, 3, 50139 Firenze, Italy
}

\begin{abstract}
The saturated dynamics of a Single Pass Free Electron Laser is considered within a simplified mean field approach. A method is proposed to increase the size of the macro-particle, which is responsible for the oscillations of the intensity of the wave. This approach is based on the reconstruction of invariant tori of the dynamics of test particles. To this aim a dedicated control term is derived, the latter acting as a small apt perturbation of the system dynamics. Implications of these findings are discussed in relation to the optimization of the laser source. PACS : 41.60.Cr, 47.10.Df, 05.45.Gg
\end{abstract}

Key words: Free Electron Laser, Hamiltonian dynamics, chaos control

\section{Introduction}

A Free Electron Laser (FEL) generates a tunable, coherent, high power radiation. A FEL differs from conventional lasers in using a relativistic electron beam as its lasing medium. The physical mechanism responsible of the light emission and amplification is the interaction between a relativistic electron beam and a magnetostatic periodic field generated in the undulator. Due to

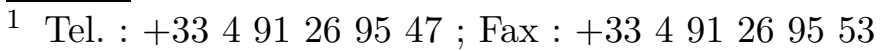

Email : bachelard@cpt.univ-mrs.fr

${ }^{2}$ Unité Mixte de Recherche (UMR 6207) du CNRS, et des universités Aix-Marseille I, Aix-Marseille II et du Sud Toulon-Var. Laboratoire affilié à la FRUMAM (FR 2291).
} 
the effect of the magnetic field, the electrons are forced to follow sinusoidal trajectories, thus emitting synchrotron radiation. This initial seed, termed spontaneous emission, is then amplified along the undulator until the laser effect is reached.

Among different schemes, Single-Pass high-gain FELs are particularly attractive since they hold the promise of resulting in flexible light sources of broad theoretical and applied interests. The coupled evolution of the radiation field and the $N$ particle beam in a Single-Pass FEL can be successfully modeled within the framework of a simplified Hamiltonian picture [1]. The $N+1$ degree of freedom Hamiltonian displays a kinetic contribution, associated with the particles, and a potential term accounting for the self-consistent coupling between the particles and the field. Hence, direct inter-particles interactions are neglected, even though an effective coupling is indirectly provided because of the interaction with the wave.

The theory predicts a linear exponential instability and a late oscillating saturation, for the amplitude of the radiation field. Inspection of the asymptotic phase-space suggests that a bunch of particles gets trapped in the resonance and forms a clump that evolves as a single macro-particle localized in space. The remaining particles are almost uniformly distributed between two oscillating boundaries, and populate the so called chaotic sea.

This observation allowed to derive a simplified Hamiltonian model to characterize the saturated evolution of the laser. Such reduced formulation consists in only four degrees of freedom, namely the wave, the macro-particle and the two boundaries, delimiting the portion of space occupied by the the uniform halo surrounding the inner bulk $[2,3]$.

Furthermore, the macro-particle rotates around a well defined fixed point and this microscopic dynamics is shown to be responsible for the macroscopic oscillations observed at the intensity level. It can be therefore hypothesized that a significant reduction in the intensity fluctuations can be gained by implementing a dedicated control strategy, aimed at confining the macro-particle in space. In addition, the size of the macro-particle is directly related to the bunching parameter, a quantity of paramount importance in FEL context [3].

Smith et al [4] showed that a test-wave, being characterized by a frequency close to the one of the wave, can effectively destroy the macro-particle and activate a consequent detrapping process. Dimonte et al [5] implemented this approach on a Travelling Wave Tube and detected a significant reduction of the intensity oscillations, followed, however, by an undesired systematic collapse of its mean-value.

In this paper, we focus on the macro-particle, spontaneously established as a result of the wave-particle interaction process in the saturated regime. In par- 

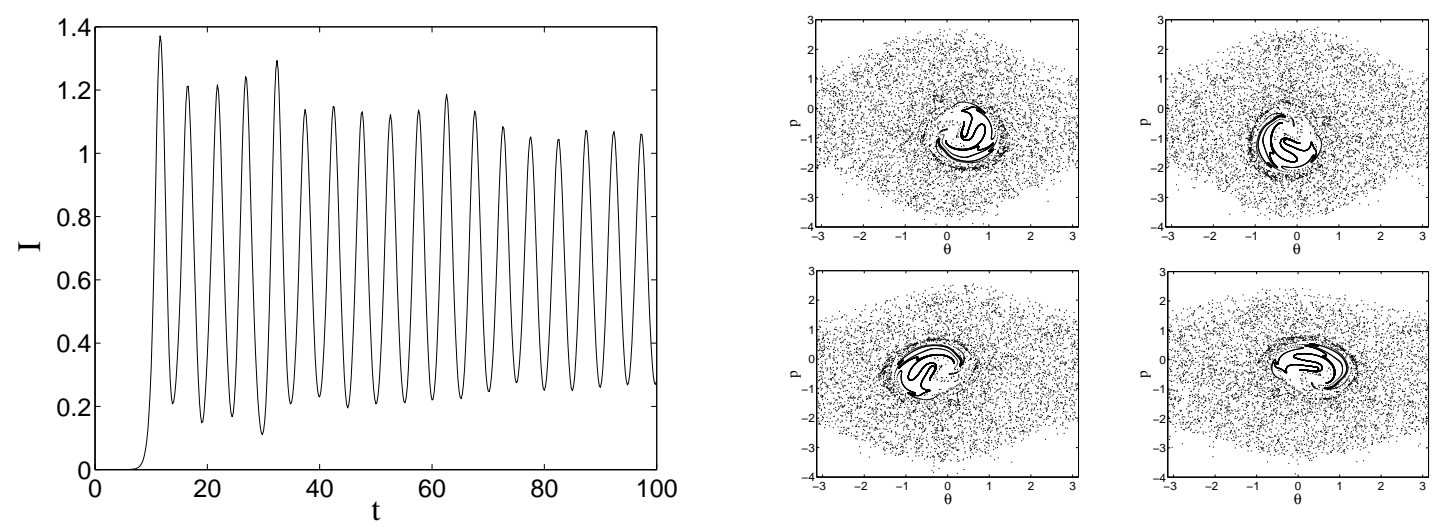

Figure 1. Left : Normalized intensity of the FEL's radiation simulated from Hamiltonian (1). Right : $N=10000$ electrons in phase space, at $t=400,401.25,402.5$ and 403.75 .

ticular we develop a dedicated technique to influence and, possibly, control its evolution, thus opening up the perspective of defining innovative approaches aiming at stabilizing the laser signal. To this end, we consider a test-particle Hamiltonian in a mean-field approach and calculate a small but appropriate control term which acts as a perturbation. The latter is shown to induce an increase in size of the macro-particle. A regularization of the dynamics is also observed, as confirmed by the reconstruction of invariant tori around the massive core.

The paper is organized as follows. In Sec. 1, we introduce the mean-field model and shortly outline its derivation from the original $N$-body Hamiltonian. In Sec. 2, a test-particle is "controlled", through the reconstruction of invariant tori of its dynamics. Finally, we draw our conclusion and discuss possible implication of the present analysis.

\section{The mean-field model}

As previously anticipated, the dynamics of a Single-Pass FEL is successfully captured by the following Hamiltonian [1] :

$$
H_{N}=\sum_{i=1}^{N}\left[\frac{p_{i}^{2}}{2}-2 \sqrt{\frac{I}{N}} \cos \left(\theta_{i}+\phi\right)\right],
$$

where $\left(\theta_{i}, p_{i}\right)$ are the position and momentum of the $i$-th particle and $(\phi, I)$ stand respectively for the phase and intensity of the radiation. In the meanfield model, the conjugated variables $(\phi, I)$ are replaced by two functions of time $\phi(t)$ and $I(t)$, obtained from direct simultations of the self-consistent dynamics. In other words, this amounts to formally neglecting the action of the electrons on the field. 
The $N$-body Hamiltonian (1) can therefore be mapped into

$$
\tilde{H}_{N}=\sum_{i=1}^{N} H_{1 p}\left(\theta_{i}, p_{i}, t\right)
$$

where

$$
H_{1 p}(\theta, p, t)=\frac{p^{2}}{2}-2 \sqrt{\frac{I(t)}{N}} \cos (\theta+\phi(t)) .
$$

Hence, the dynamics of a FEL can be adressed by studying the evolution of a test particle, obeying Hamiltonian (2). The functions $I(t)$ and $\phi(t)$ act as external fields and are here imposed by assuming their simplified asymptotic behaviour as obtained by a frequency analysis [6] :

$$
2 \sqrt{\frac{I(t)}{N}} e^{i \phi(t)} \approx F-\epsilon \sum_{k=1}^{K} W_{k} e^{i \omega_{k} t}
$$

in the reference frame of the wave.

\section{Hamiltonian control of a test-particle}

The Hamiltonian control addresses systems which are close to integrable, i.e. whose Hamiltonian can be written as $H=H_{0}+V$, where $H_{0}$ is integrable and $V$ a perturbation of order $\epsilon$ (compared to $H_{0}$ ). The results we use here have been proven rigorously $[7,8]$. In practice, it can be shown that a suitable control term $f$ of order $\epsilon^{2}$ exists such that $H_{0}+V+f$ has an invariant torus at a given frequency $\omega_{0}$. In our case, the perturbation corresponds to the oscillating part of the intensity. The interaction term of Hamiltonian (2) reads

$$
2 \sqrt{\frac{I(t)}{N}} \cos (\theta-\phi(t))=F \cos \theta-\epsilon \operatorname{Re}\left(\mathrm{e}^{\mathrm{i} \theta} \mathrm{W}(\mathrm{t})\right) .
$$

Thus, our integrable Hamiltonian can be cast in the form

$$
H_{0}=\frac{p^{2}}{2}-F \cos \theta
$$

whereas the time-dependent perturbation $V$ is identified as

$$
V(t, \theta)=\epsilon \operatorname{Re}\left(\mathrm{e}^{\mathrm{i} \theta} \mathrm{W}(\mathrm{t})\right) .
$$

First, we express Hamiltonian (3) into action-angle variables $(\varphi, J)[9]$. Then, we expand $H_{0}$ around $J=J_{0}$, which in turn identifies the region where the invariant torus is reconstructed in :

$$
H_{0}(J)=E_{0}+\omega_{0}\left(J-J_{0}\right)+\delta\left(J-J_{0}\right)^{2}+O\left(\left(J-J_{0}\right)^{3}\right) .
$$


Likewise, the $\theta$-component of perturbation (4) is expanded as

$$
e^{i \theta}=\sum_{m=0}^{M} \sum_{n=-L}^{L} \alpha_{m, n}\left(J-J_{0}\right)^{m} e^{i n \varphi}+O\left(\left(J-J_{0}\right)^{M+1}\right)
$$

The control term reads [8]

$$
f(\varphi, t)=V\left(J_{0}, \varphi, t\right)-V\left(J_{0}-\Gamma \partial_{\varphi} V\left(J_{0}, \varphi, t\right), \varphi, t\right),
$$

where $\Gamma$ is a linear operator acting on an element of the Fourier basis as :

$$
\Gamma e^{i(\omega t+n \varphi)}=\frac{e^{i(\omega t+n \varphi)}}{i\left(\omega+n \omega_{0}\right)} .
$$

At the second order in $\epsilon$ (which is the main term), the control term reads

$$
f(\varphi, t)=\epsilon^{2} w(\varphi, t) \Gamma \partial_{\varphi} v(\varphi, t)-\epsilon^{2} \delta\left(\Gamma \partial_{\varphi} v(\varphi, t)\right)^{2},
$$

where $v$ and $w$ are the first terms in the expansion of $V$ :

$$
V(J, \varphi, t)=v(\varphi, t)+\left(J-J_{0}\right) w(\varphi, t)++O\left(\left(J-J_{0}\right)^{2}\right),
$$

which gives

$$
\Gamma \partial_{\varphi} v(\varphi, t)=\sum_{k=0}^{K} \sum_{n=-L}^{L} \frac{n \alpha_{0, n} W_{k}}{\omega_{0} n+\omega_{k}} e^{i\left(n \varphi+\omega_{k} t\right)},
$$

and

$$
w(\varphi, t)=\sum_{k=0}^{K} \sum_{n=-L}^{L} \alpha_{1, n} W_{k} e^{i\left(n \varphi+\omega_{k} t\right)}
$$

where $W_{k}$ are the Fourier coefficients of $W$ :

$$
W(t)=\sum_{k=0}^{K} W_{k} e^{i \omega_{k} t}
$$

Numerically, two Fourier modes are considered for $W(t)(K=2)$, and eleven for the $\theta$-dependent part of the perturbation $(4)(L=5)$. The perturbation is of amplitude $\epsilon=1 / 5$. The expansion is performed around $J_{0} \approx 1.33$ (which corresponds to the energy $\left.E_{0}=0\right)$.

In action-angle variables, the regularization of the dynamics (see Fig.2) is clearly seen. Rather than a single torus, the control term generates a continuous set of invariant tori, thus expanding the regular domain in phase space.

Unfortunately, the exact change of variables from $(\varphi, J)$ to $(\theta, p)[9]$ has a singularity at the pendulum separatrices. In order to implement our control on the whole space, we use a simplified, but regular, change of variables which 

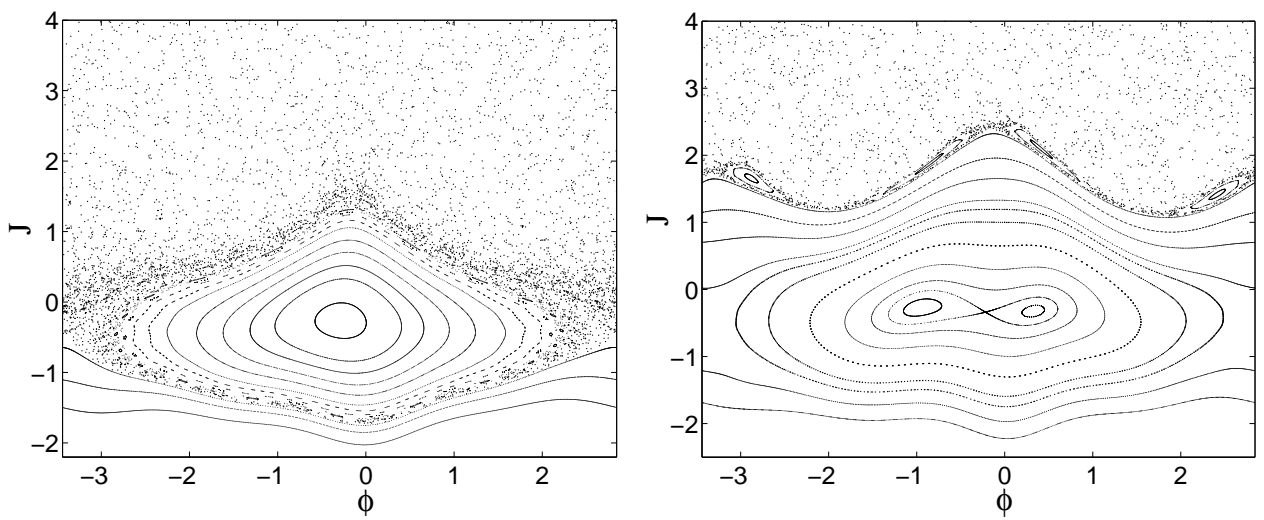

Figure 2. Poincaré sections of a test-particle of Hamiltonian $H_{0}(J)+V(J, \varphi, t)$ (left) and $H_{0}(J)+V(J, \varphi, t)+f(\varphi, t)$ (right), in the pendulum action-angle variables.
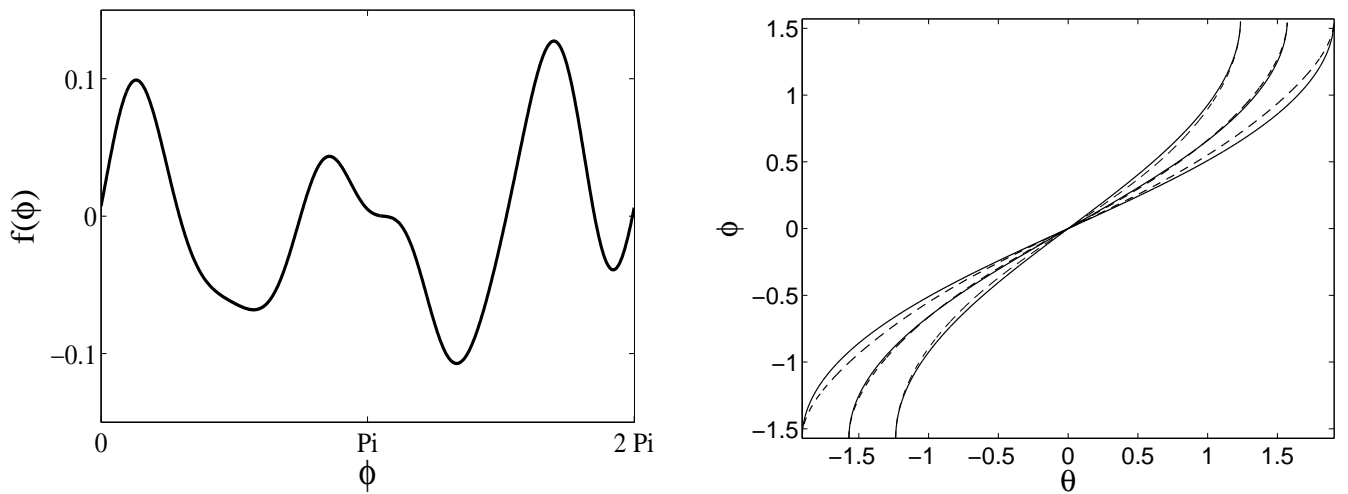

Figure 3. Left : control term $f(\phi, t)$, at $t=0$. Right : comparison of the exact and simplified changes of variables : $\varphi$ is plotted as a function of $\theta$, for a given energy (and so for a given $J$ ). The plain line corresponds to the exact change, the dotted to the simplified one. The curves correspond, from bottom left to bottom right, to $E=0.5,0$ and -0.5 .

mimics the exact one in the region of the invariant torus predicted by the control (the error in this region is less than 4\%) (see Fig.3) :

$$
\tan \varphi=\frac{\theta}{p}
$$

In terms of the $(\theta, p)$ variables, our control term now reads :

$$
\tilde{f}(\theta, p, t)=f\left(\arctan \frac{\theta}{p}, t\right)
$$

and its regularity is now the one of the function $(\theta, p) \mapsto \arctan \frac{\theta}{p}$. Therefore, the controlled dynamics of a test-particle is given by Hamiltonian :

$$
H_{c}(\theta, p, t)=H_{1 p}(\theta, p, t)+\tilde{f}(\theta, p, t) .
$$



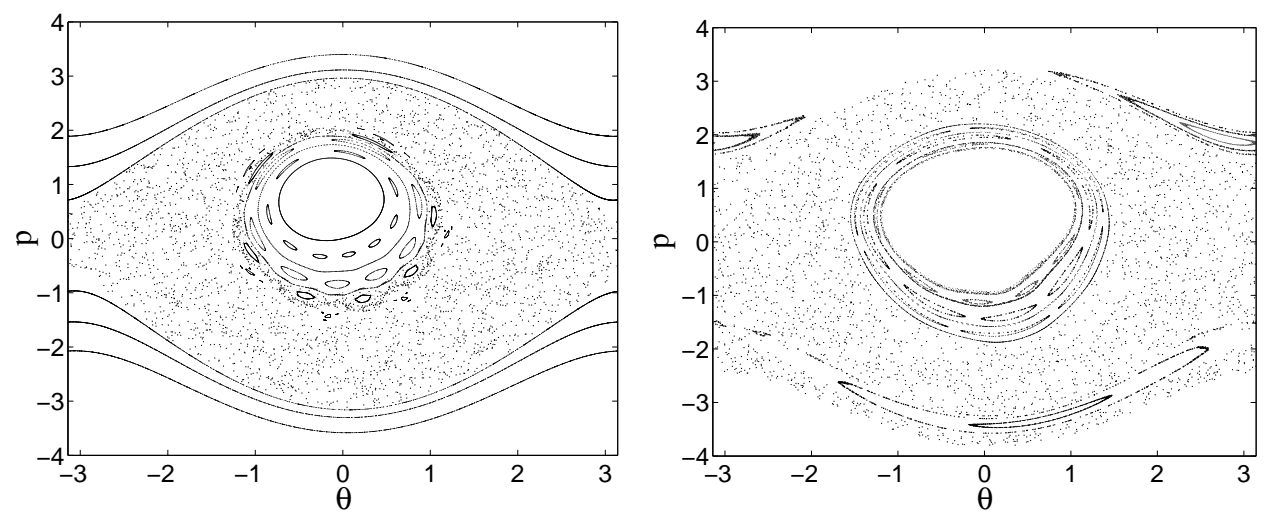

Figure 4. Poincaré sections of a test-particle of Hamiltonian $H_{1 p}(\theta, p, t)$ (left) and $H_{1 p}(\theta, p, t)+\tilde{f}(\theta, p, t)$ (right).

In the $(\theta, p)$ variables (see Fig.4), the control is successful in reconstructing some invariant tori around the macro-particle. In other words, it enlarges this regular structure.

\section{Conclusion}

In this paper, we considered a simplified mean-field approach to investigate the saturated dynamics of a Single Pass FEL. In particular, we showed that the size of the macro-particle can be increased by adding a small pertubation to the system, thus resulting in a low cost correction in term of energy. The main idea is to build invariant tori localized at specific positions : this method is utterly general and could be succesfully used to adjust the size of the macroparticle, thus possibly enhancing the bunching factor. Analogously, by limiting the portion of phase-space spanned by the macro-particle, one could aim at stabilizing the laser signal. Two future lines of investigation are foreseen. First, we intend to implement the computed control term in the framework of the original $N$-body self-consistent picture and explore possible beneficial effects for the evolution of the radiation field. Further, it is planned to apply the above technique to the case of the reduced Hamiltonian of $[2,3]$. We point out that an experimental test of the control method on a modified Travelling Wave Tube has been done [10] in absence of self-consistency.

\section{References}

[1] Bonifacio, R., et al. (1990). Physics of the high-gain FEL and superradiance. Rivista del Nuovo Cimento, 3, pp. 1-69. 
[2] Tennyson, J.L., J.D. Meiss, and P.J. Morrison (1994). Self-Consistent Chaos in the Beam-Plasma Instability. Physica D, 71, pp. 1-17.

[3] Antoniazzi, A., Y. Elskens, D. Fanelli and S. Ruffo (2006). Statistical mechanics and Vlasov equation allows for a simplified Hamiltonian description of SinglePass Free Electron Laser saturated dynamics, Europ. Phys. J. B, in press.

[4] Smith, G.R. and N.R. Pereira (1978). Phase-locked particle motion in a largeamplitude plasma wave. Phys. Fluids, 21, pp. 2253-2262.

[5] Dimonte, G., J.H. Malmberg (1977). Destruction of Trapped-Particle Ocillations. Phys. Rev. Lett., 38, pp. 401-404.

[6] Laskar, J. (1999). Introduction to Frequency Map Analysis. Proc. of NATO ASI Hamiltonian Systems with Three or More Degrees of Freedom, (C. Simò Ed, Kluwer), pp. 134-150.

[7] Vittot, M. (2004). Perturbation theory and control in classical or quantum mechanics by an inversion formula. J. Phys. A: Math. Gen., 37, pp. 6337-6357.

[8] Chandre C., M. Vittot, G. Ciraolo, Ph. Ghendrih, R. Lima (2006). Control of stochasticity in magnetic field lines. Nuclear Fusion, 46, pp. 33-45.

[9] Lichtenberg, A.J. and M.A. Libermann (1983). Regular and Stochastic Motion. Springer, New York.

[10] Chandre, C., G. Ciraolo, F. Doveil, R. Lima, A. Macor and M. Vittot (2005). Channeling chaos by building barriers. Phys. Rev. Lett., 74, 074101. 